\title{
INTERNAL FRICTION AND VISCOSITY ASSOCIATED WITH MOBILE INTERSTITIALS IN THE PRESENCE OF A KINK HARMONICALLY OR UNIFORMLY MOVING IN ANISOTROPIC BODY-CENTERED CUBIC METALS
}

\author{
T.ö. Ogurtani and A. Seeger \\ Max-Planck-Institut fur Metalfforschung, Institut fir Physik, Stuttgart, \\ F.R.G. \\ and Middle East Technical University, Ankara, Turkey
}

\begin{abstract}
The power dissipation due to mobile octahedral interstitials in the stress field of a harmonically oscillating or uniformly moving kink of a dislocation line is derived for anisotropic BCC metals using the discrete Fourier transformation of the elastic Green's function. The viscosity and the drag force acting on the kink moving uniformly along the dislocation line are formulated which shows strong velocity and the temperature dependent behaviour.
\end{abstract}

1. Introduction.- In a previous paper /1/ the present authors have successfuliy developed a unified linear-response theory of internal friction due to movements of interstitial impurities in the presence of periodically time dependent but spatiaily inhomogeneous stress field excitations in terms of discrete $\underline{k}$-space Fourier transformation for a BCC lattice. This specific problem of energy dissipation is closely related to the atomic migration of point defects in time dependent, spatially fluctuating fields which has been studied extensively by Seeger and Hornung $/ 2 /$ and Hornung /3/ using the conventional Fourier transformation in time and space. In a more recent theoretical investigation by Ogurtani and Seeger $/ 4 /$, this problem is reformulated using the discrete Fourier k-space transformation with respect to space and supplemented by a Laplace transformation with respect to time. Also, the physical scope of the problem is enlarged by considering the hopping motion with defect reactions between two or more non-equivalent sites for an arbitrary lattice structure. The desired explicit expression for the Fourier transform of the "partial concentration" of the interstitial impurities at each energetically distinquishable sites is obtained, and later is employed in the energy dissipation calculation.

2. Power Dissipation in Inhomogeneous Fields.- The power input $I(t)$ for the selected super-cell sample may be written in the following form $/ 1,4 /$ which is valid even for a nonlinear response system:

$$
I(t)={ }_{i=1}^{3} \prod_{i} \quad \sum_{\underline{k}}^{\sum} \varepsilon V_{k} \underline{\tilde{U}}_{\underline{k}}^{*}(t) \cdot \partial_{t} \underline{\tilde{c}}_{\underline{k}}(t),
$$

where $N_{1}, N_{2}$, and $N_{3}$ are even integer numbers representing the size of the sampling domain, and $v_{k}$ is the corresponding vector set in the reciprocal lattice space $/ 1 /$. 
Here, $\tilde{\tilde{U}}_{k}(t)$ are the discrete Fourier $\underset{\sim}{k}$-space transforms of the kink-interstitial interaction energy fields, and $\tilde{c}_{k}(t)$ are the partial concentrations of octahedral interstitial atoms at the three types of interstitial sites in a $\mathrm{BCC}$ lattice in the discrete Fourier k-space representation. The partial concentrations denoted by a vectorial quantity which has three components according to the three different tetragonality axes. We denote the octahedral sites with axes parallel to [100], [010], or [001] by the subscript 1,2 or 3 , respectively. As far as the time dependence of the interaction energy field is concerned, we deal with two different but similar cases in this paper:

and

$$
\underline{\tilde{u}}_{\underline{k}}^{u}(t)=\underline{\tilde{u}}_{\underline{k}}^{S}+\underline{\tilde{u}}_{\underline{k}}^{D} \exp \left(i \omega_{\underline{k}} t\right)
$$

$$
\underline{\tilde{u}}_{\underline{k}}^{h}(t)=\underline{\tilde{u}}_{\underline{k}}^{S}+\underline{\tilde{u}}_{\underline{k}}^{D}\{\exp (i \omega t)+c \cdot c \cdot\} / 2,
$$

where $\tilde{u}_{k}^{S}$ corresponds to the static part (bias) of the field, and $\underline{\tilde{u}}_{k}^{D}$ is related to the amplitude of the dynamic component of the excitation (induced or external). Expression (2) indicates that the individual Fourier components have their own oscillation frequency denoted by $\omega_{k}$ (dispersion), which will be the case for a kink moving rigidiy and uniformly along the dislocation line. Relationship (3), on the other hand, corresponds to an inhomogeneous field which has simple harmonic oscillations in time, with an arbitrary frequency, $\omega$, about a static field or bias, $\underline{\tilde{u}}_{k}^{S}$. The partial concentrations related to these fields can be written down using the general theory advocated by the present authors $/ 1,4 /$ in the following formats, respectively:

$$
\tilde{c}_{\underline{k}}^{u}(t)=\left(c^{0} / 6 k_{B} T\right) \sum_{\underline{k}, i} \frac{-P_{i}(\underline{k}) \exp \left(i \omega_{k} t\right)}{P_{i}(\underline{k})+i \omega_{k}} B^{i}(k) \cdot \underline{\tilde{u}}_{\underline{k}}^{D}+\underline{c}_{\underline{k}}(\infty),
$$

and

$$
\tilde{c}_{\underline{k}}(t)=\left(c^{0} / 6 k_{B} T\right) \sum_{\underline{k}, i}\left\{\frac{-P_{j}(\underline{k}) \exp (i \omega t)}{P_{i}(\underline{k})+i \omega}+c \cdot c \cdot\right\} \underline{B} \underline{\underline{i}}(\underline{k}) \cdot \underline{\tilde{u}}_{\underline{k}}^{D}+\underline{\tilde{c}}_{\underline{k}}(\infty),
$$

where $\tilde{c}_{k}(\infty)$ is the thermodynamic equilibrium concentration in $\underline{k}$-space which is closely rélated to the static part of the interaction field. In the above expressions (4) and (5), $P_{i}(\underline{k})$ are all real positive numbers in the closed interval $\{0,2\}$, zero occurs only when $\underline{k}=0$ and $i=1$, (acoustic branch $/ 1 /$ ), the $P_{i}(\underline{k})$ correspond to the inverse relaxation time spectrum which is normalized wuth respect to $4 \Gamma$, where $\Gamma$ is the atomic jump frequency of interstitials. $C^{0}$ is the initial, uniform, total concentration of the interstitial species in the matrix. $\underline{B}^{i}(\underline{k})$ is a dyadic uniquely determined by the crystal structure in the present case (ño chemical reaction).

If one uses the steady-state solutions given by, eqs. (4) and (5), which clearly indicate that the system has a linear response, together with the fundamental power relationship (1), the following can be obtained for the loss or dissipation part $/ 1,5 /$ : 


$$
I_{D}^{u}(t)=-\left(C^{0} / 12 k_{B} T\right) \prod_{j=1}^{3} N_{j} \sum_{\underline{k}, i}^{\sum} \frac{\omega_{k}^{2} P_{j}(\underline{k})}{P_{j}^{2}(\underline{k})+\omega_{k}^{2}} \underline{u}_{\underline{k}}^{D^{*}} \cdot \underline{B^{i}} \underline{\underline{k})} \cdot \underline{\tilde{u}_{k}^{D}},
$$

where we have assumed that $\omega_{k}=-\omega_{-k}$ which is the case for a kink moving with a constant velocity along the dislocation line. For a harmonic oscillation in time, a similar expression may be obtained from eqs. (1) and (5) where one should replace $\omega_{k}$ by win eq. (6). The energy dissipation per cycle for a harmonic system can be calculated from the following expression:

$$
\Delta W^{h}=\int_{0}^{2 \pi / \omega} I^{h}(t) d t=\int_{0}^{2 \pi / \omega} I_{D}^{h}(t) d t,
$$

which yields immediately:

$$
\Delta W^{h}=-\pi\left(C_{T}^{0} / 6 k_{B} T\right) \quad \sum_{\underline{k}, i} \quad \frac{\omega \tau_{i}(\underline{k})}{1+{ }^{2} \tau_{i}^{2}(\underline{k})} \quad \underline{\tilde{u}}_{\underline{k}}^{D} \cdot \underline{B}^{i}(\underline{k}) \cdot \underline{\tilde{u}}_{\underline{k}}^{D^{*}},
$$

where $\tau_{i}(\underline{k})$ is the relaxation time associated with the $i^{\text {"th }}$ branch of the spectrum, given by $\tau_{i}(\underline{k})=1 / P_{i}(\underline{k})$. Also, $C_{T}^{0}=C^{0} \Pi N_{i}$. As shown in reference $/ 4 /$, the relaxation time spectrum is affected by the presence of the static field, and it can be calculated using the first order perturbation theory.

3. The Elastic Dipole Interaction.- Actually the general dissipation and storage relationship deduced in this paper (see /5/) is valid regardless of the nature of the interaction energy field, e.g. dipole, quadrupole, stress, magnetic, electric, etc.

In the case of stress field interactions, one can write the following relationship using the elastic dipole approximation $/ 5,6 /$

$$
\begin{aligned}
\underline{\tilde{u}}_{\underline{k}}^{D} \cdot \underline{B}^{i}(\underline{k}) \cdot \underline{\tilde{u}}_{\underline{k}}^{D *}= & v_{A}^{2} \lambda_{2}^{2}\left\{|\operatorname{Tr}(\underline{\tilde{\sigma}}(\underline{k}))|^{2}{ }^{1}{ }_{q}{ }^{1} q^{\prime} B_{q q^{\prime}}^{i}(\underline{k})\right. \\
& \left.+2 \eta \operatorname{Tr}(\underline{\tilde{\sigma}}(\underline{k})) 1_{q} B_{q m}^{i}(\underline{k}) \tilde{\sigma}_{m m}(\underline{k})+\eta^{2} \tilde{\sigma}_{m m}(\underline{k}) B_{m m^{\prime}}^{i} \tilde{\sigma}_{m^{\prime} m^{\prime}}(\underline{k})\right\},
\end{aligned}
$$

where $n=\left(\lambda_{1}-\lambda_{2}\right) / \lambda_{2}$, which is closely related to the shape factor of the point defect. $1_{q}$ is the summation operator with respect to dummy indices $q$. In above equation, $v_{A}$ is the atomic volume, $\lambda_{1}$ and $\lambda_{2}$ are the principal values of the tetragonal elastic dipole tensor, and $\tilde{\sigma}(\underline{k}, t)$ is the discrete Fourier transform of the interacting stress tensor. Eq. (9) clearly indicates that in the calculation of the energy dissipation and the power consumption, only the diagonal matrix elements of the stress tensor in the principal-axis representation are important. This is due to the fact that the elastic dipole tensor associated with the octahedral or tetrahedral interstitial sites in a $B C C$ structure is completely diagonalized in the principal-axis representation. 
4. The Stress Field of a Kinked Dislocation Line.- The discrete Fourier transform of the stress field associated with a dislocation 1 ine in an infinite, homogeneous and anistropic linear elastic continuum may be written down as /5/

$$
\tilde{\sigma}_{j k}(\underline{k}, t)=c_{j k m n} c_{\ell p t s} b_{\ell}\left(\underline{i}_{n} \cdot \underline{k} \underline{k} \cdot \underline{i}_{s}\right) \tilde{G}_{t m}(\underline{k}) f e^{-i \underline{k} \cdot \underline{r}^{\prime}} d S_{p}^{\prime} \text {, }
$$

where $i_{n}$ is the unit Cartesian vector set and $C_{j k m n}$ are the elastic stiffnesses. $\tilde{G}_{t m}(k)$ is the discrete Fourier transform of the elastic Green's function, which can be calculated from the conventional Fourier integral transform of the elastic Green's function as defined by Dederichs and Leibfried /7/. We want to underline the fact that the integral in eq. (10) is the only term which depends upon the configuration of dislocation line in its slip plane because the surface integral is bounded by the dislocation line itself. Any time dependent perturbations of the dislocation 7 ine will be reflected in the Fourier transform of the stress tensor by the surface integral term, $s(\underline{k}, t)$. Therefore, it is valid to call $s(\underline{k}, t)$ is the dynamic form factor of a planar curvilinear dislocation line (quasi-static approximation).

5. The Dynamic Form Factor Associated With the Kinked Dislocation Performing Uniform or Harmonic Motions.- The detailed calculations of the dynamic form factor for various dislocation configurations, static, oscillating harmonically with time or moving uniformly and rigidly, are presented elsewhere /5,8/. The exact expression for the dynamic form factor for a kink moving harmonically along the dislocation line as follows:

$$
s^{h}(\underline{k}, t)=\frac{i a \sin \left(\underline{k} \cdot \underline{r}_{0}\right)}{\underline{k} \cdot \underline{t}\left(\underline{k} \cdot \underline{r}_{0}\right)} \exp \left(-i \underline{k} \cdot \underline{t} A_{k} \sin \omega t\right)+s^{(+)}(\underline{k}),
$$

where $s^{(+)}(\underline{k})$ is the form factor for a straight dislocation lying along the $+a / 2$ Peierly valley $/ 5 /$. The expression (11) shows the generation of the higher harmonics in the Fourier transform of the stress field. However, when the kink oscillation amplitude, $A_{K}$, is small compared to the projection of the wave length along the dislocation line, the following linear approximation can be performed:

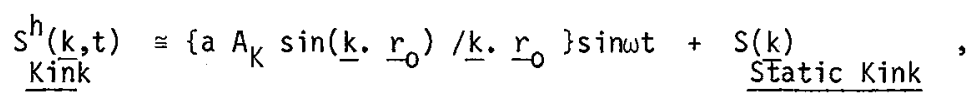

where "a" is the distance between two nearest Peierls valley, and $\underline{t}$ is a unit tangent vector along the straight dislocation (screw or edge). Similarly, $\underline{r}_{0}=\left(\underline{a}+W_{K} t\right) / 2$, where $W_{K}$ is the kink width along the dislocation line.

The dynamical form factor for a kink moving with a constant velocity $\underline{v}_{K}$ along the dislocation can be calculated /5/ which resulty

$$
S_{\underline{k i n k}}^{u}(\underline{k}, t)=\frac{i a \sin \left(\underline{k} \cdot \underline{r}_{0}\right)}{-\underline{k} \cdot \underline{t}\left(\underline{k} \cdot \underline{r}_{0}\right)} \exp \left(-i \omega_{\underline{k}}^{k} t\right)+s^{(t)}(\underline{k}),
$$

where $\omega_{\underline{k}}^{K}=\underline{k} \cdot \underline{v}_{K}$, which shows that oscillation frequencies of the individual Fourier 
components depend upon the wave vector $k$. This is typical acoustic dispersion behavior for elastic wave propagation in the medium.

6. The Viscosity and the Drag Force Acting on a Kink Moving Uniformly Along the Dislocation Line.- The kink viscosity due to interstitial defects can be obtained as a scalar quantity from the following operational relationship

$$
I_{D}^{u}(t)=-\mu_{K} v_{K}^{2}
$$

which according to eq.(6), (9), (10) and (13) results:

$$
\begin{aligned}
& \mu_{K}\left(V_{K}\right)=\left(c^{0} a^{2} \lambda_{2}{ }^{2} / 12 k_{B} T\right)\left(\prod_{m=1}^{3} N_{m}\right) \sum_{k, i} \tau_{i}(\underline{k}) /\left\{1+(\underline{k} \cdot \underline{t}) V_{K}^{2} \tau_{i}^{2}(\underline{k})\right\} \\
& \times \frac{\sin ^{2}\left(\underline{k} \cdot \underline{r}_{0}\right)}{\left(\underline{k} \cdot \underline{r}_{0}\right)^{2}}\left\{\mid \operatorname{Tr}\left(\left.\underline{\tilde{Q}}(\hat{k})\right|^{2}\left|1_{q} e_{q, i}^{0}(\underline{k})\right|^{2}\right.\right. \\
& +2 n \operatorname{Tr}(\underline{\underline{Q}}(\hat{k})) 1_{q} e_{q, i}^{0^{*}}(\underline{k}) e_{n, i}^{0}(\underline{k}) \tilde{Q}_{n n}(\hat{k}) \\
& \left.+n^{2}\left|e_{m, i}^{0}(\underline{k}) \tilde{Q}_{m m}(\hat{k})\right|^{2}\right\},
\end{aligned}
$$

where $\tilde{Q}_{j k}(k)=c_{j k m n} \quad c_{\text {lpts }} n_{p} b_{\ell} k_{n} k_{s} \tilde{g}_{t m}(k)$, and $\tilde{g}_{t m}=\underline{k}^{2} \tilde{G}_{t m}(k)$. In above expression $\hat{k}$ is the unit vector in the direction of $\underline{k}$, and $\underline{e}^{0}, i(k)$ corresponds to the orthogonalized set of eigen unit vectors associated with the interstitial's hopping matrix $/ 1 /$, where $\underline{B}^{i}(\underline{k}) \equiv\left|\underline{e}_{,}^{0}, i^{>} \underline{e}^{0}, i\right|$. Similarly, the viscous drag force, $F_{K}$ acting on the moving kink with uniform velocity and rigidly along the dislocation line can be obtained from the following expression:

$$
\underline{F}_{K}=-\mu_{K}\left(v_{K}\right) \underline{v}_{K} \text {. }
$$

7. The Internal Friction Enhancement Due to Sma11 Amplitude Kink Oscillation.- The energy dissipation per cycle can be obtained from eqs.(8), (9), (10) and (12) as follows:

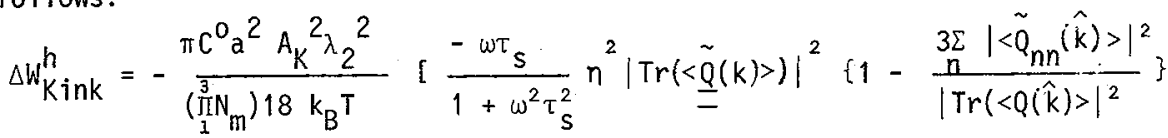

$$
\begin{aligned}
& +\sum_{\frac{k}{i} \neq 0} \frac{\sin ^{2}\left(\underline{k} \cdot \underline{r}_{0}\right)}{\left(\underline{k} \cdot \underline{r}_{0}\right)^{2}} \frac{\omega \tau_{i}(\underline{k})}{1+\omega^{2} \tau_{i}^{2}(\underline{k})} 3\left\{|\operatorname{Tr}(\underline{\tilde{Q}}(k))|^{2}\left|1_{m} e_{m, i}^{0}(\underline{k})\right|^{2}\right. \\
& \left.\left.+2 n \operatorname{Tr}(\underline{\tilde{Q}} \hat{(k)}) 1_{q} e_{q, i}^{0^{*}}(\underline{k}) e_{n, i}^{0}(\underline{k}) \tilde{Q}_{n n}(\hat{k})+n^{2}\left|e_{m, i}^{0 *}(\underline{k}) \tilde{Q}_{m m}(\hat{k})\right|^{2}\right\}\right]
\end{aligned}
$$

where $\tilde{\sigma}_{j, k}(\underline{k}, t) \stackrel{\text { def. }}{=} S(\underline{k}, t) \tilde{Q}_{j, k}(\hat{k})$, and $<>$ denotes the angular averaging process. 
8. Discussion.- Without making any extensive computer simulation studies which will be presented elsewhere, the following conclusions can be easily reached: a). The energy dissipation is directly proportional to the mean concentration of interstitials $c^{0}$, which is assumed to be uniformly distributed initially. b). The induced internal friction strength depends quadratically on both the amplitude of the kink oscillation and the intensity factor of the elastic dipole tensor. c). The interstitials which can be represented by a simple isotropic dilation $n=0$, can still cause internal friction and viscous drag due to the inhomogeneity of the stress field which is characterized by a non-vanishing trace of the Fourier transform of the stress tensor. d). Also, one can show rigorously that $\operatorname{Tr}(\langle\underline{Q}(k)>)$ is identically zero for non-sessile dislocation configurations /5/. Therefore, for an isotropic BCC metal the induced Snoek peak (uniform modes), represented by the first term in eq.(17) is completely missing. However, one can still get an appreciable amount of energy dissipation in the vicinity of the pure Snoek relaxation $\left(\tau_{s}\right)$ frequency via optical relaxation modes $(i=2,3)$ for the non-vanishing $k$ vectors. The later contributions, however, are very sensitive to the amplitude modulation factor through the kink width, $W_{K}$. As one might anticipate, the greater the kink width, the smaller the spectral broadening of the internal friction peak (induced), again due to the modulation factor, $\sin { }^{2}\left(\underline{k} \cdot \underline{r}_{0}\right) /\left(\underline{k} \cdot \underline{r}_{0}\right)^{2}$, which is calculated for various kink widths in reference $/ 5 /$.

We can state that one might get a considerable amount of power dissipation related to the internal friction or viscous drag from the mobile (even isotropic) interstitials if one has relatively narrow kink oscillating or moving uniformly along the pure screw dislocation. The situation for $71^{\circ}$ mixed dislocations is more favorable as far as these isotropic point defects are concerned for which power dissipation still takes place even for very wide geometrical kinks. As a final remark the authors want to state very clearly that one can have an observable amount of viscosity or internal friction enhancement due to mobile isotropic defects in the presence of kinked screw dislocations, even for large kink widths, as long as the metal shows a high degree of elastic anisotropy, which is the case for most metals and alloys in practice.

\section{REFERENCES}

1. T. 0. Ogurtani and A. Seeger, "The Internal Friction Associated with Mobile Octahedral Interstitials in the Presence of Inhomogeneous Fields in BCC Metals", J. Appl. Phys., (tentative issue: 1 July 1983).

2. A. Seeger and W. Hornung, Int. Friction and Ultrasonic Attenuation in Crystalline Solids (D. Lenz and K. Lücke, ed.) Springer, Berlin, Vol.1, 222 (1975).

3. W. Hornung, Phys. Stat. Sol.(b) 54, 341 (1972).

4. T. 0. Ogurtani and A. Seeger, "The Kinetics of Diffusion of. Interstitials with Chemical reactions in Arbitrary Time Dependent Inhomogeneous Fields", J. Chem. Phys., (tentative issue: 15 November 4983 ).

5. T. O. Ogurtani and A. Seeger, "Internal Friction and Viscosity Associated with Mobile Interstitials in the Presence of a Kink Moving Harmonically or Uniformly in Anisotropic BCC Metals", to be published in J. Appl. Phys. (1983).

6. E. Kröner, Kontimuumstheorie der Versetzungen und Eigenspannungen, Springer,Berlin, (1958).

7. P. Dederichs and G. Leibfried, Phys. Rev., Vol. 188, 1175 (1969).

8. A. D. Brailsford, J. App1. Phys., Vol. 43, 1380 (1972). 